\title{
Prediction of Heart Disease Severity Measurment Using Deep Learning Techniques
}

\author{
Rina S. Patil ${ }^{\mathrm{a}, 1}$ and Dr. Mohit Gangwar ${ }^{\mathrm{b}}$ \\ ${ }^{a}$ Ph.D. Research Scholar, Department of Computer Science and Engineering, Bhabha University, \\ Bhopal, Madhya Pradesh, India \\ ${ }^{b}$ Dean-Engineering, Bhabha University, Bhopal, Madhya Pradesh, India
}

\begin{abstract}
Machine learning enables AI and is used in data analytics to overcome many challenges. Machine learning was the growing method of predicting outcomes based on existing data. The computer learns characteristics from the test implementation, then applies characteristics to an unknown dataset to predict the result. Classification is an essential technique of machine learning which is widely used for forecasting. Some classification techniques predict with adequate accuracy, while others show a small precision. This research investigates a process called machine learning classification, which combines different classifiers to enhance the precision of weak architectures. Experimentation using this tool was conducted using a database on heart disease. The collecting and measuring data method were designed to decide how to use the ensemble methodology to improve predictive accuracy in cardiovascular disease. This paper aims not only to enhance the precision of poor different classifiers but also to apply the algorithm with a neural network to demonstrate its usefulness in predicting disease in its earliest stages. The study results show that various classification algorithmic strategies, such as support vector machines, successfully improve the forecasting ability of poor classifiers and show satisfactory success in recognizing heart attack risk. Using ML classification, a cumulative improvement in the accuracy was obtained for poor classification models. That process efficiency was further improved with the introduction of feature extraction and selection, and the findings show substantial improvements in predictive power.
\end{abstract}

Keywords. Heart disease, Machine learning, Internet of Things, Feature extraction, Feature selection

\section{Introducion}

Insufficient asset status of tribal communities and unawareness are the critical problems in the rural healthcare system. For example, 650 million people live in a rural area Bharat to tell the story 33 per day with this financial benefit. It will be difficult for a private individual to access innovative medical facilities to have associate degree access. There is, therefore, a great need to build an outsized health monitoring system that is low-cost, economical, and easy to use. Here since we would like to provide medical assistance to even the poorest segment of society, the emphasis on low prices may not be tasteful. Exhaustive research has been devoted to researching various

${ }^{1}$ Rina S. Patil, Ph.D. Research Scholar, Department of Computer Science and Engineering, Bhabha University Email. rinapatil190@gmail.com. 
developments, such as advances in knowledge in supplementation and strengthening established medical facilities. The Internet of Things (IoT) has generally been linked to interface open therapeutic assets. It offers older adults and clinicians any persistent, secure, viable, and keen medical services management. Indeed, before making the IoT vision a fact, many test protection problems must be tended to. We need to address essential questions about further empowering the IoT while maintaining all aspects, such as faith, protection, and security. We have guided this work to assist people who are keen on growth and improvement in this arena. Various overviews have just been suggested, but they rely for the most part upon a broader vision that involves "Things"located, "Web"-arranged, and "Semantic"-arranged thoughts, or on a layered sight. Our vision remains a good decision for fundamental leadership, regardless of the limits of its hypothetical meticulousness, because we are speaking about the system-level mission.

\section{Literature Survey}

According to the authors [1], cardiac disease is one of the world's leading causes of death, and precipitate disease prediction is essential. The computer-aided machines assist the doctor as a gimmick in detecting and treating heart disease. This paper aims to broaden cardiovascular disease related to the heart and briefly explore open decision support systems through data mining and smart hybrid techniques for computing and researching heart disease. With many methodologies, a lot of DSS remains to be projected on heart disease.

Explain that according to estimates, heart disease is one of the most fundamental reasons behind deaths worldwide [2]. In challenging scientific support systems, data mining techniques are prevalent and can identify concealed patterns and associations in medical data. Data mining classification methods have so far been used to test the different forms of heart-based issues. This essay aims to build a data mining techniques framework using classification methods of analysis mining. The various clustering methods, nearest neighbor, EM, and the most restricted hypothesis methodology for first heart disease are discussed in this article.

Using different machine learning algorithms in [3] [4], the multi-classification problem has been solved. Both systems deal with massive unstructured data and continue to use the methodology of supervised learning. The results obtained showed that classification accuracy was higher than conventional machine learning algorithms.

According to Chengjin Yu. et al. [5], an adversarial training approach is proposed to multitask learning to estimate multi-type Cardiac Indices in MRI and CT. By using multitask learning networks, these task dependencies are shared and learned. Lastly, they transferred parameters learned from MRI to CT. A series of experiments were performed. The authors first optimized system efficiency over 2900 cardiac MRI images through ten-fold cross-validation. Then the network was run on an independent data set with 2360 cardiac CT images. The results of all experiments on the proposed reverse mapping indicate excellent performance in estimating multiple cardiac indices.

\section{Propsoed System Design}

The framework first gathers the input sequence states of each device, then uses ADC to transform it from analog - to - digital. when the transformation has been completed, it 
will be received by the microcontroller and saved in the database same moment. The dynamic monitoring method parallels work for entire application events and displays them to the front wave user interface. Then, the suggested machine learning algorithms operate in the system's central ware, often checks all model parameters from the target threshold, if any time account level below minimum confidence and max resistance, then the output devices are automatically executed. Simultaneously, as the system calculates the hazardous amount of the operation state, the program also means the time count with the same state, and the timer and GPS messaging system will run whenever it reaches the expected to meet.

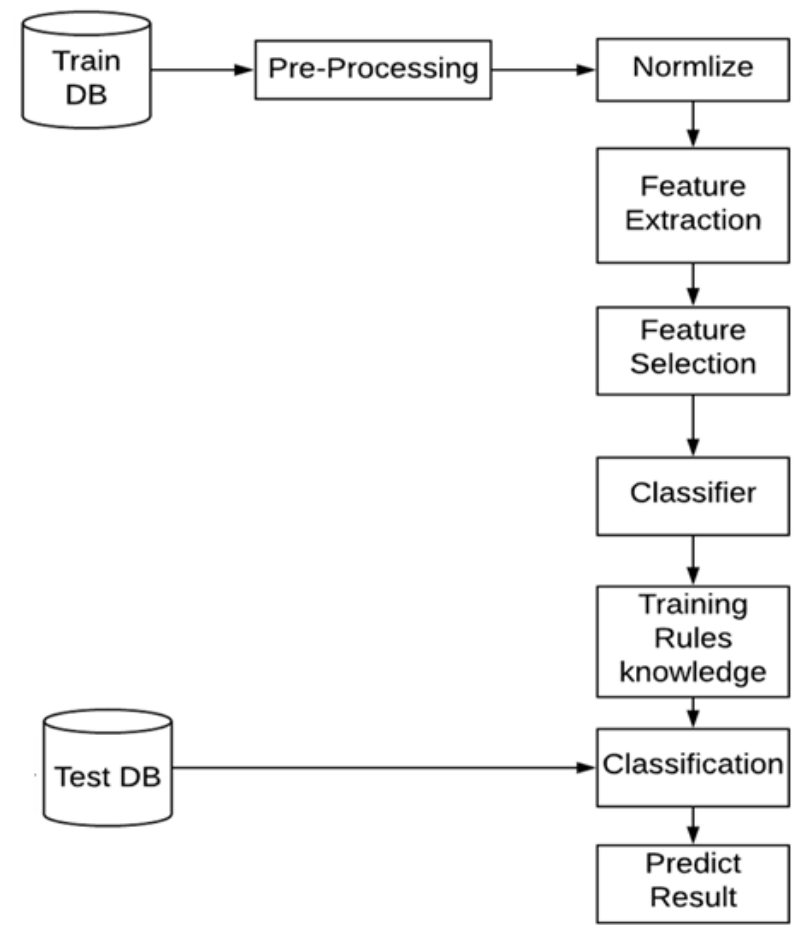

Figüre 1. Proposed system architecture

\section{Results and Discussions}

With different Java and Mobile and web platforms, the proposed architecture has been transmitted. The Figure 2 below shows that the recognized part causes data loss and ECG sensor (it may be useful for loss and increased rate sensors). In contrast, stress dramatically increases the number of proposed devices that can be used all the time for the given packet size. Here X-axis shows the no. of users, and Y shows the average packet loss from various IoT devices below, Figure 2 shows the system accuracy with no experimental test analyses. 


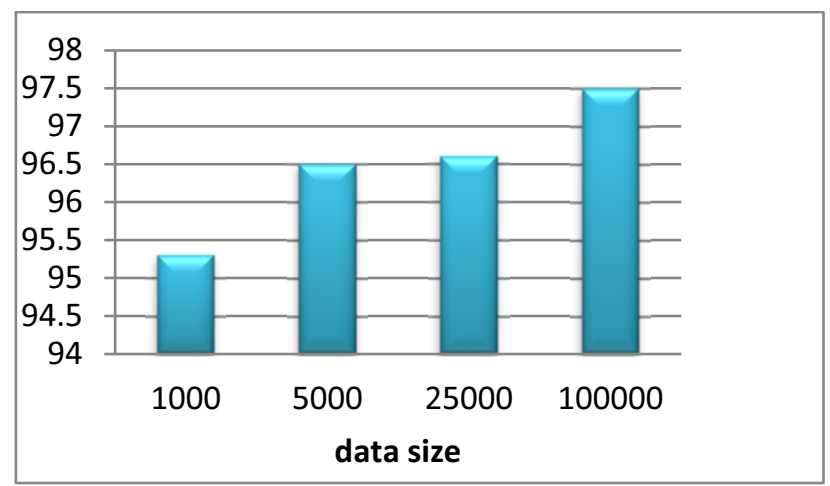

Figure 2. System accuracy of proposed system with false ratio.

Figure 3 provides the thorough overview of the next experimental investigation to measure the effectiveness of the proposed method.

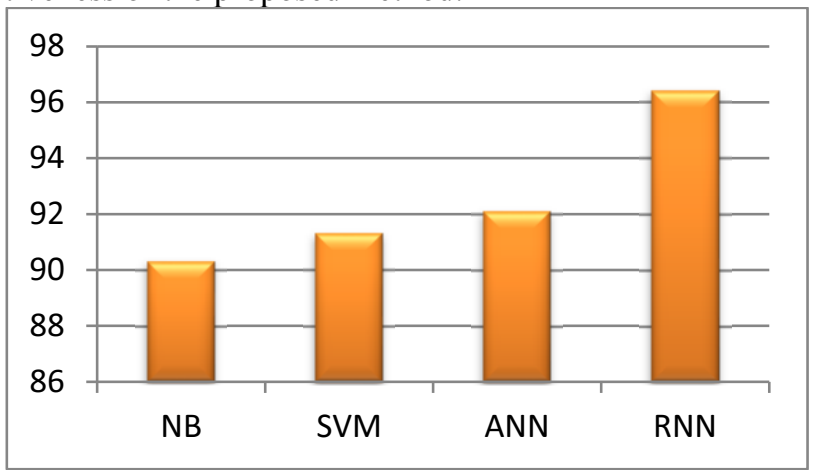

Figure 3. System accuracy of proposed system with various existing systems.

The overall experimental analysis describes a comparative analysis of proposed system vs various existing systems. The system supports Real-time health tracking and disease predictions over the network. Detection probability is more substantial than different learning styles, and it can function based on synthetic and real-time datasets. The device also has the option to convey alarm when there is some criticality over the internet.

\section{Conclusions}

In this research, we introduced an ideal framework for prediction models in real-time, and users with the coronary disease can use that. Unlike many other systems, it is capable of tracking and predicting both. The system's diagnostic method will predict cardiovascular disease utilizing ML algorithms, and the predictive conclusions are based on the dataset example of heart disease. On the other hand, the device is very economical. we used an enthused pulse sensor and sent the data via the Arduino suite microcontroller to mobile devices. To check the variances and raise the alarm if the patient's heart rate increases above the usual heart rate. In order to prove the system's efficacy, we performed tests with both the tracking and diagnostic method. We conducted experiments with supervised machine classification methods such as NB, SVM and ANN. The procedure was done with the validation set test, and $96 \%$ efficacy 
of the proposed method was obtained with the Random Forest. We performed two tests for the monitoring system. We conducted with various healthy patients on the first sample, and we interacted with 20 individuals with a cardinal illness on the second. In both cases, the control system's accuracy was $100 \%$. We intend to use the proposed device in the future and to omit by use of a sensor.

\section{References}

[1]. Vijayashree, J., and N. Ch Sriman Narayana Iyengar. "Heart disease prediction system using data mining and hybrid intelligent techniques. A review." Int. J. Bio-Sci. Biotechnol 8 (2016). 139-148

[2]. Singla, Meenu, and Kawaljeet Singh. "Heart Disease Prediction System using Data Mining Clustering Techniques."

[3]. B.Pandu Ranga Raju, B.Vijaya Lakshmi, C.V.Lakshmi Narayana, Detection of Multi-Class Website URLs Using Machine Learning Algorithms, International Journal of Advanced Trends in Computer Science and Engineering, pp. 1704-1712,Volume 9, No.2, 2020.

[4]. Roobaea Alroobaea, An Empirical combination of Machine Learning models to Enhance author profiling performance, International Journal of Advanced Trends in Computer Science and Engineering, pp. 2130-2137, Volume 9, No.2, 2020.

[5]. Sun, Yingnan, Frank P-W. Lo, and Benny Lo. "A deep learning approach on gender and age recognition using a single inertial sensor." 2019 IEEE 16th International Conference on Wearable and Implantable Body Sensor Networks (BSN). IEEE, 2019.

[6]. Lo, Frank P-W., et al. "A novel vision-based approach for the dietary assessment using deep learning view synthesis." 2019 IEEE 16th International Conference on Wearable and Implantable Body Sensor Networks (BSN). IEEE, 2019.

[7]. Uddin, Md Zia, and Mohammad Mehedi Hassan. "Activity recognition for cognitive assistance using body sensors data and deep convolutional neural network." IEEE Sensors Journal 19.19 (2018). 84138419 .

[8]. M.Ganesan and Dr.N.Sivakumar, "IoT based heart disease prediction and diagnosis model for healthcare using machine learning models ", Proceedings of International Conference on Systems computation automation and networking 2019, IEEE- 978-17281-1524.

[9]. AKM Jahangir Alam Majumder, Yosuf Amr ElSaadany, Roger Young Jr., and Donald R. Ucci, "An Energy Efficient Wearable Smart IoT System to Predict Cardiac Arrest", Hindawi Advances in Human-Computer Interaction Volume 2019, Article ID 1507465, 21 pages.

[10]. C. Yu et al., "Multitask Learning for Estimating Multitype Cardiac Indices in MRI and CT Based on Adversarial Reverse Mapping," in IEEE Transactions on Neural Networks and Learning Systems, DOI. 10.1109/TNNLS.2020.2984955

[11]. Liu, X., Faes L., Kale A et al. A comparison of deep learning performance against healthcare professionals in detecting diseases from medical imaging. $9^{*}$ a systematic review and meta-analysis, Vol 1(6), 271-297, 2019.

[12]. Byrne, N., Velasco Forte, M., Tandon, A., Valverde, I., \& Hussain, T.. A systematic review of image segmentation methodology, used in the additive manufacture of patient-specific 3D printed models of the cardiovascular system, JRSM Cardiovascular Disease, 2016, https.//doi.org/10.1177/2048004016645467

[13]. Damen Johanna A A G, Hooft Lotty, Schuit Ewoud, Debray Thomas P A, Collins Gary S, Tzoulaki Ioanna et al. Prediction models for cardiovascular disease risk in the general population. systematic review, 2016, BMJ 2016; 353. i2416.

[14]. Matthew Marsh A, Literature Review of Image Segmentation Techniques and Matting for the Purpose of Implementing "Grab-Cut", Department of Computer Science at Rhodes University, South Africa, Available http.//citeseerx.ist.psu.edu/viewdoc/download?doi=10.1.1.103.1334\&rep=rep1\&type=pdf

[15]. Mamta Sharma, Farheen Khan, Vishnupriya Ravichandran, "Comparing Data Mining Techniques Used for Heart Disease Prediction, f Engineering and Technology, vol.5, Issue 6, 2017 